Corrigendum

\title{
Corrigendum to "Neuroprotective Effects of the Sonic Hedgehog Signaling Pathway in Ischemic Injury through Promotion of Synaptic and Neuronal Health"
}

\author{
Sen Yin, ${ }^{1,2}$ Xuemei Bai, ${ }^{2}$ Danqing Xin, ${ }^{2}$ Tingting Li, ${ }^{2}$ Xili Chu, ${ }^{2}$ Hongfei Ke, ${ }^{2}$ Min Han, \\ Wenqiang Chen, ${ }^{1}$ Xingang $\mathrm{Li} \odot{ }^{\circ},{ }^{1}$ and Zhen Wang $\oplus^{2}$ \\ ${ }^{1}$ Qilu Hospital, Cheeloo College of Medicine, Shandong University, Jinan, Shandong, China \\ ${ }^{2}$ Department of Physiology, School of Basic Medical Sciences, Cheeloo College of Medicine, Shandong University, Jinan, \\ Shandong 250012, China \\ Correspondence should be addressed to Xingang Li; lixg@sdu.edu.cn and Zhen Wang; wangzhen@sdu.edu.cn \\ Received 5 May 2021; Accepted 5 May 2021; Published 26 May 2021
}

Copyright (C) 2021 Sen Yin et al. This is an open access article distributed under the Creative Commons Attribution License, which permits unrestricted use, distribution, and reproduction in any medium, provided the original work is properly cited.

In the article titled "Neuroprotective Effects of the Sonic Hedgehog Signaling Pathway in Ischemic Injury through Promotion of Synaptic and Neuronal Health" [1], the authors have identified that in Figure 4, the incorrect images were provided due to an error during the preparation of the manuscript. The authors confirm that this error does not affect the results of the article and the corrected Figure 4 is shown below. 

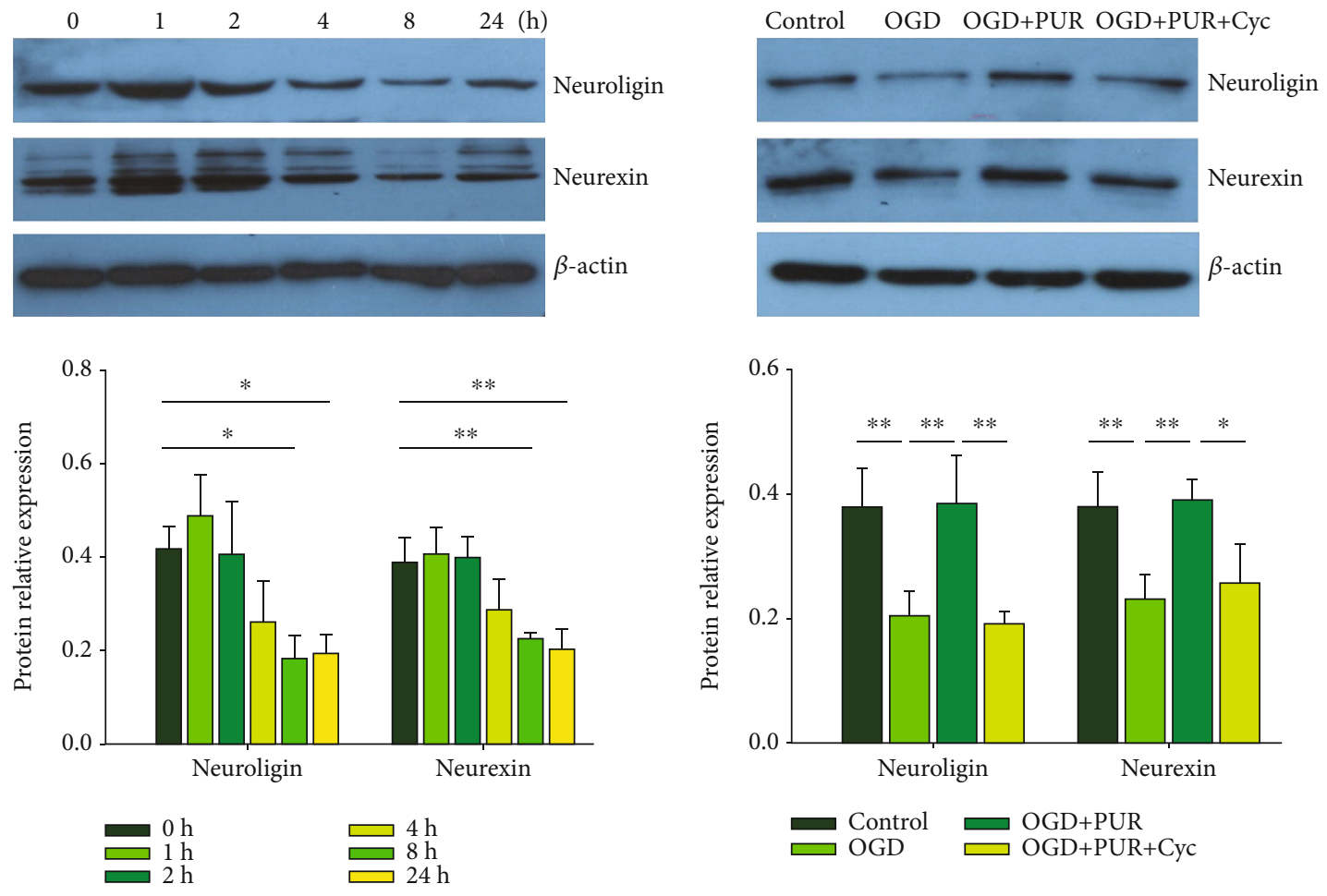

(a)

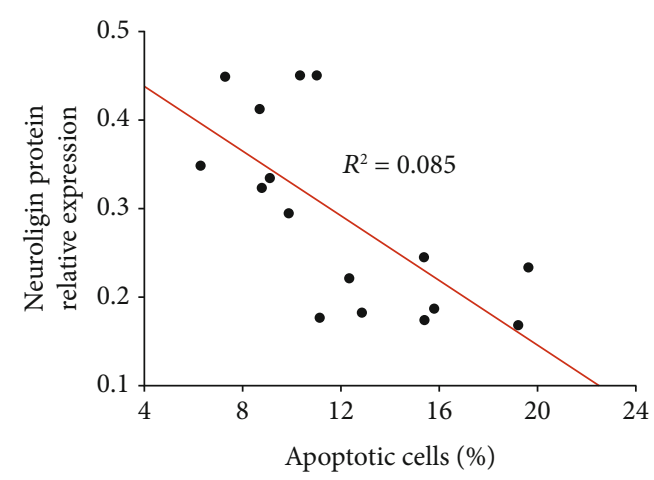

(b)

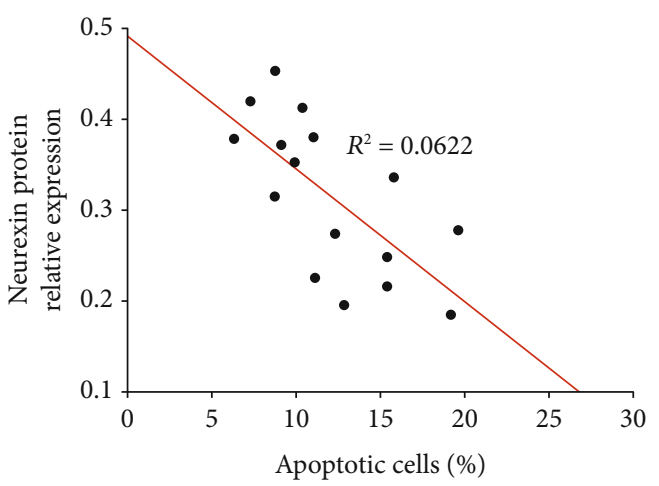

(c)

FIGURE 4: PUR activation of Shh on OGD-induced neuroligin and neurexin: (a) protein levels of neuroligin and neurexin at 1,2, 4, 8, and 24 h after OGD as determined by Western blot ( $N=3$ /group); (b) protein levels of neuroligin and neurexin at $24 \mathrm{~h}$ after OGD as determined by Western blot $(N=4$ /group); (c) Pearson correlation coefficients obtained between neuroligin/neurexin expressions and apoptosis following PUR treatment. Values represent the mean $\pm \mathrm{SD} ;{ }^{*} p<0.05$ and ${ }^{* *} p<0.01$ according to ANOVA with the Dunnett test in (a) and Tukey's post hoc comparisons in (b).

\section{References}

[1] S. Yin, X. Bai, D. Xin et al., "Neuroprotective Effects of the Sonic Hedgehog Signaling Pathway in Ischemic Injury through Promotion of Synaptic and Neuronal Health," Neural Plasticity, vol. 2020, Article ID 8815195, 11 pages, 2020. 